\title{
The Influence of Ozone Forcing on Blocking in the Southern Hemisphre
}

A.LELA

Taihoro Nukurangi

1 University of Canterbury, Christchurch, New Zealand, ${ }^{2}$ National Institute of Water and Atmospheric Research, Lauder, New Zealand

\section{Introduction}

Atmospheric blocking refers to large, quasi-stationary, high pressure features that persist beyond the synoptic time scale and inhibit the midlatitude zonal flow. Most of the blocking in the Southern Hemisphere occurs in the South Pacific region and, to a lesser extent, the Southwestern Atlantic and exhibits a strong seasonal cycle with blocking shown to have significant affects on the weather over mid-latitude continental regions.

In this study, we use the persistent positive anomaly (PPA) approach to indentify blocking events. A grid point is considered to be blocked if the anomaly in $500 \mathrm{hPa}$ geopotential height $(\mathrm{GPH})$ exceeds $100 \mathrm{~m}$ and lasts
for more than 5 days.

\section{Model}

We use the NIWA-UKCA coupled atmosphere-ocean-chemistry climate model (AOCCM). In order to reveal the influence of ozone we compare eference runs ("REF') in which the model is forced by the A1 scenario (Where ozone depleting substances (ODSs) follow historical emisions beros ceasing in accordance with the Montreal protocol), to sensitivity ("'fODS"). For comparison we also use runs in which greenhouse gases are held fixed at 1960's levels ("fGHG").

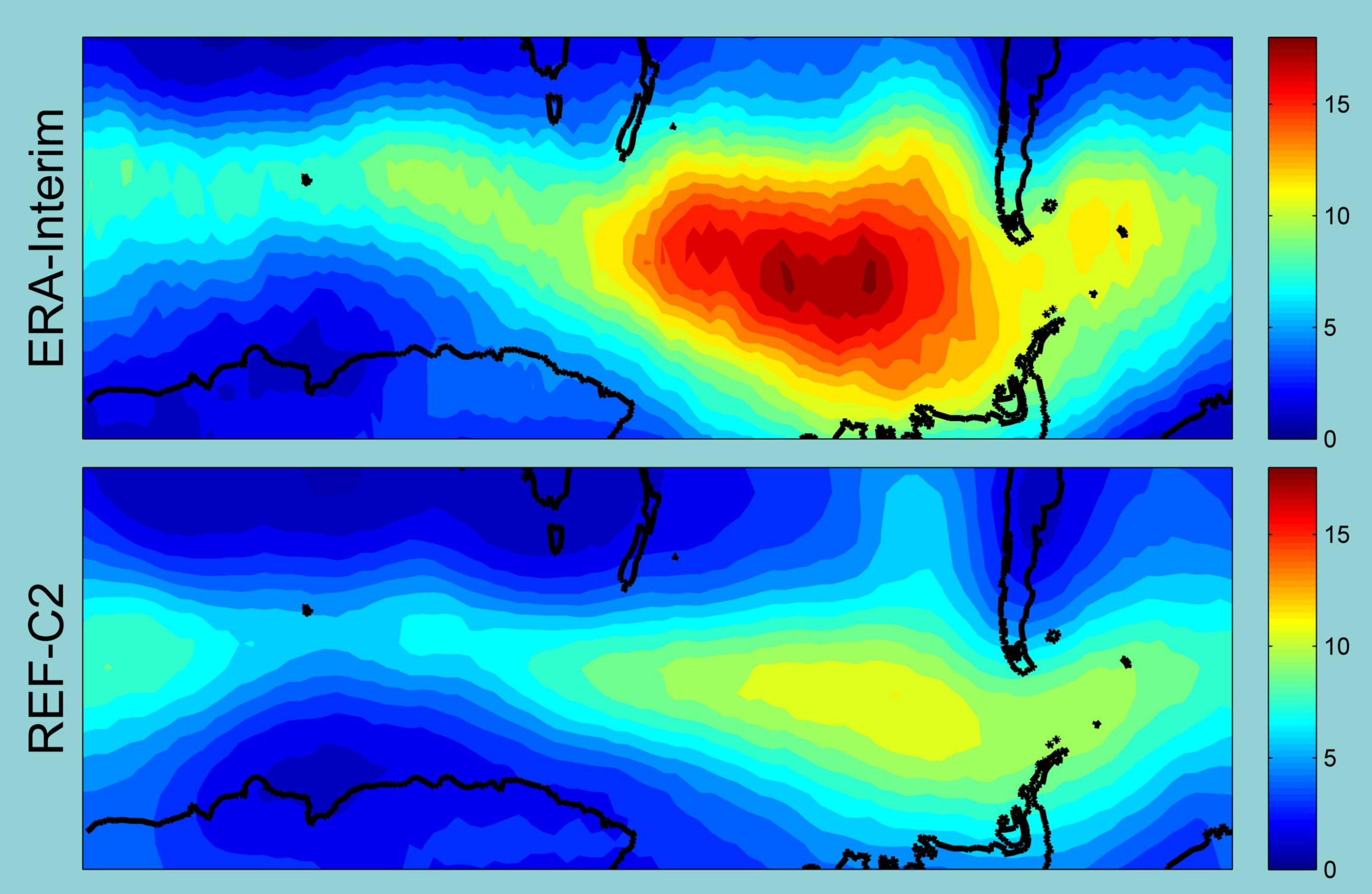

In order to validate the model we examine the annual mean blocking frequency for the period 1979-2014, shown in the above figure for both the ERA-Interim reanalysis (top) and the REF ensemble (bottom). The model does well in simulating the spatial pattern, correctly positioning the maxima in blocking frequency in the Eastern Pacilc sector and the minima in the Indian Ocean sector. The spatial correlation between these patterns is 0.88 . However, the model underestimates the magnitude of the blocking frequency is a common problem amongst climate models

Acknowledgements:
This research has b

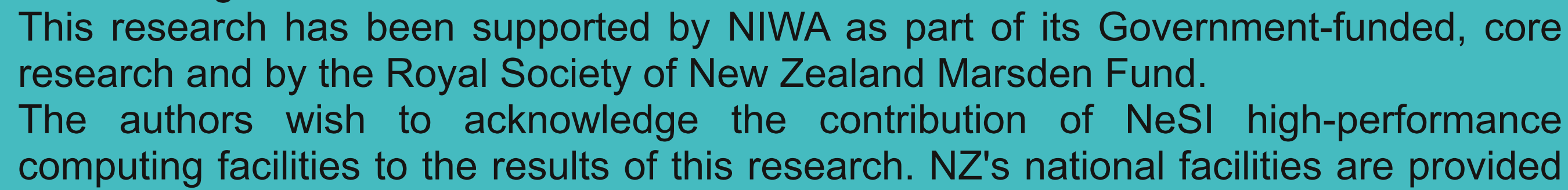

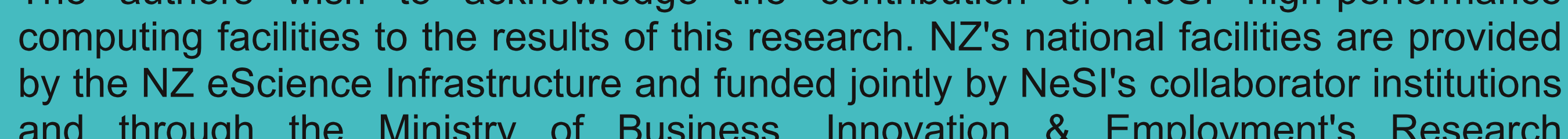
and through the Ministry of Business, Illovintion
Infrastructure programme. URL hitps://www.nesi. org.nz

\section{Influence of Ozone}

In order to highlight the effect of ozone depletion, we initially examine the period 1987-2036 which corresponds to the period of maximum ozone depletion as simulated by the model. During this period the October mean total column ozone south of $70^{\circ} \mathrm{S}$ is less than 220 Dobson Units. The top row of the figure below shows the seasonal blocking frequencies in the REF runs. There is a large seasonal cycle with blocking more frequent during the winter season. The second row of the figure illustrates the effect of Ozone depletion by showing the differences between the REF and fODS ensembles in each season. depletion appears to have affected the blocking frequency: (1) In the South Atlantic in summer blocking appears to have increased as a result of ozone depletion. (2) In the Southern Indian Ocean in winter (and to a lesser extent in spring) blocking has decreased. The South Atlantic effect is the larger of the two and our a priori expectation is that ozone depletion generally affects the summer season. For these reasons we will focus on the Atlantic region during summer. In contrast to the South Atlantic, modelled blocking in the South Pacific is not affected by ozone depletion. By contrasting these two regions we will investigate the reasons behind the change in the Atlantic region, the regions are highlighted by green boxes in the figure below.
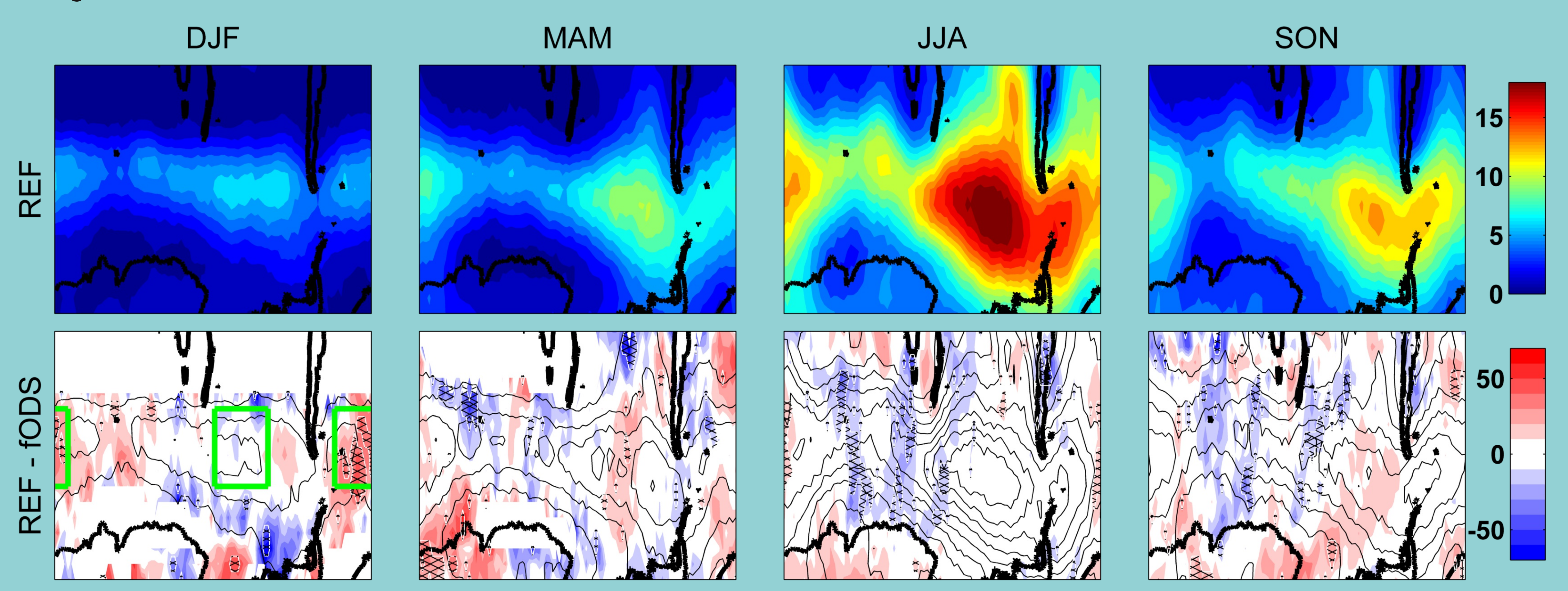

The figure to the right shows the time series of the summer blocking frequency over the Atlantic and Pacific regions for the three scenarios and the ERA-Interim reanalysis. The ERA-Interim trends in blocking frequency in each of the regions match the modelled trends, namely a positive trend in the South Atlantic and no significant trend in the South Pacific. blocking has two clear trends, increasing up until about 2010 with a slower decrease thereafter. The timing of this change might be expected from an ozone driven effect as ozone depletion peaks at around that time before slowly recovering over the remainder of the 21 st century. A similar pattern is also observed for the fGHG time series. In contrast to the Atlantic, the Pacific region shows no significan differences between the REF and fODS runs

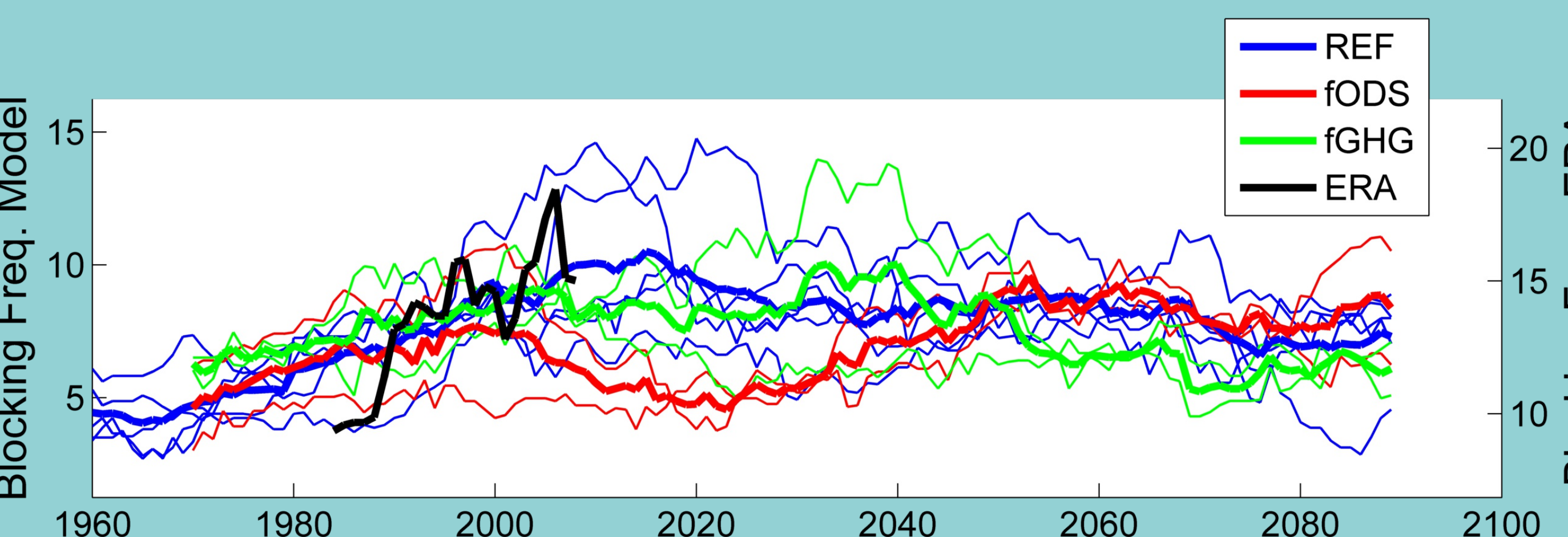
over the course of the simulations.

\section{SAM Composite}

The Southern Annular Mode (SAM) is the major mode of variability in the extra-tropical Southern Hemisphere; ozone depletion and recovery is known to effect stratosphere-
troposphere coupling of this mode. The following figure illustrates how this effect relates to blocking. The upper panel of the figure shows the SAM composite corresponding of the blocking event; lag 0 corresponds to the first day of the blocking event There is a positive SAM anomaly prior to the blocking event. This anomaly lasts for $40-50$ days and peaks around $30-50 \mathrm{hPa}$, but extends through much of the lower stratosphere and upper troposphere. It comes to an end abruptly at the onset of the blocking event. The contrast is clear when comparing this behavior to the equivalen structure for the Pacific region shown in the lower panel of the figure. In this case the SAM anomaly is much smalle and confined to the troposphere,

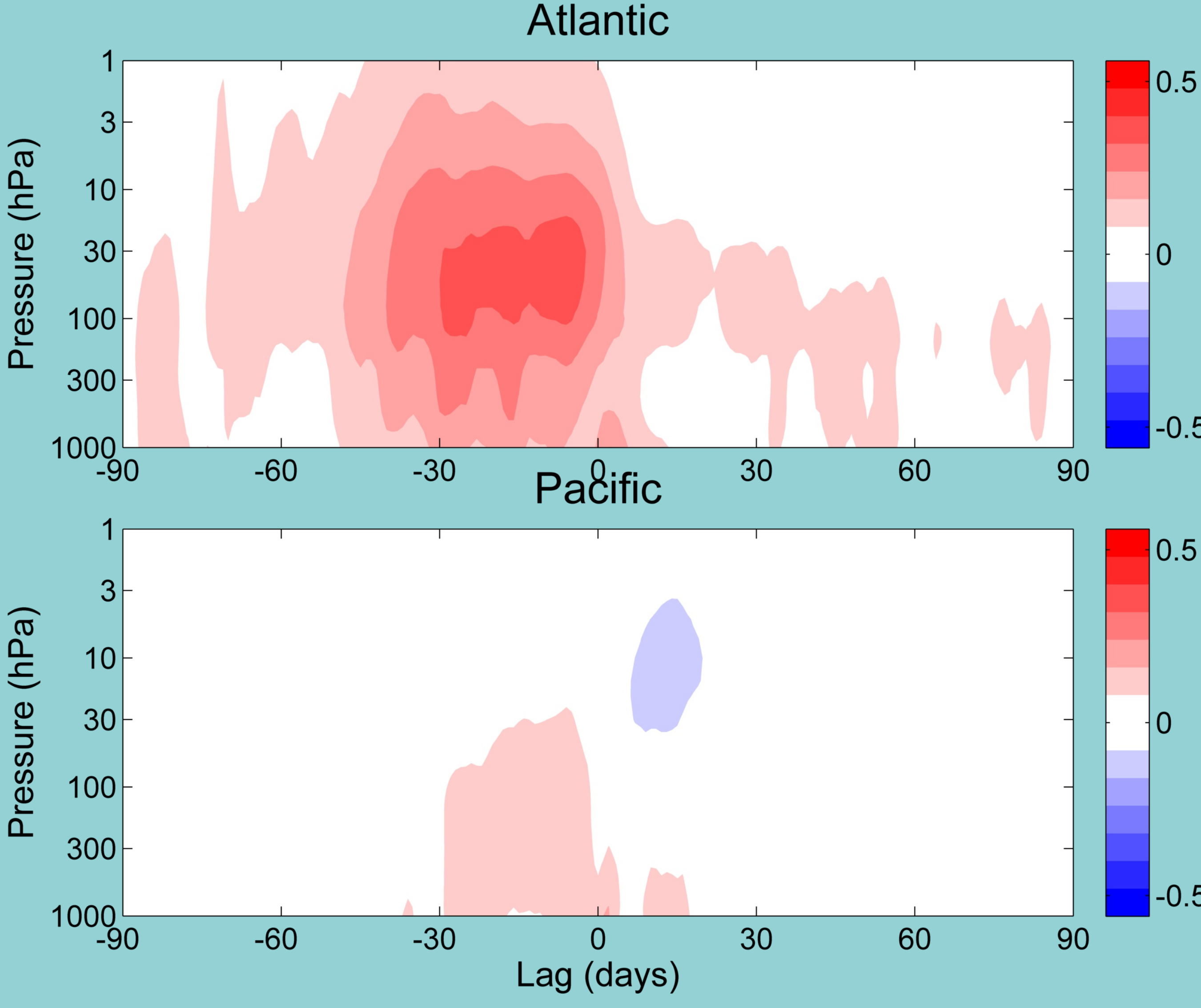

\section{Summary}

Blocking events in the South Atlantic are shown to follow stratospheric positive anomalies in the SAM index; this is no the case for South Pacific blocking events. This relationship positive SAM anomalies, leads to an increased frequency of locking in the South Allanic while having little effect in the South Pacific. Similarly, ozone recovery, having the opposite effect on the SAM, leads to a reduction in blocking frequency in the South Atlantic, although this may be somewhat counteracted by the effect of increasing GHGs.

Further detail can be found in

Dennison, F. W., Mc Donald, A. J., and Morgenstern, $O$ (2016), The Influence of Ozone Forcing on Blocking in the Southern Hemisphere, J. Geophys. Res. (in review) 